\title{
Directional sensitivity to a tactile point stimulus moving across the fingerpad
}

\author{
DAVID V. KEYSON and ADRIANUS J. M. HOUTSMA \\ Institute for Perception Research, Eindhoven, The Netherlands
}

\begin{abstract}
The ability of subjects to discriminate between directions of a point contact moving across the fingerpad was examined. Subjects were required to report, using an adaptive two-interval, twoalternative forced-choice procedure, whether in two sequential stimuli the direction of motion changed in a clockwise or counterclockwise direction. The overall mean orientation-change threshold across eight stimulus orientations was approximately $14^{\circ}$, with the lowest threshold for the point motion toward the wrist. This observed lower threshold in the distal-to-proximal direction is thought to be due to stretching of the skin at the tip of the fingernail, to which one may be particularly sensitive. For all orientations, thresholds were generally more uniform and higher than those reported on vibrotactile linear contactor arrays for horizontal and vertical orientations.
\end{abstract}

Although little research has been reported on the thresholds for tactile angle discrimination of a moving point across the skin, several studies have examined tactile and haptic perception of orientation for static stimuli. In particular, the degree to which deviations can be detected from referent or standard vertical, horizontal, and diagonal orientations has been considered. However, a clear understanding of directional sensitivity for a single point contactor, as opposed to sensitivity for a static linear contactor array, is lacking. Furthermore, it is not clear how directional discrimination at opposite ends of the principal meridians may vary, given skin stretch by a single point. Thus the present study has two major objectives: (1) To estimate the degree to which subjects can discriminate the angle of a point contact moving across the right index finger; and (2) to determine how threshold levels vary with stimulus orientations in relation to a fixed finger position.

Studies of nonmoving tactile and haptic line orientation perception suggest that an oblique effect (Appelle, 1972) occurs in the haptic and tactile modalities, whereby the discrimination of stimuli presented along or straight across the finger axis (i.e., in a vertical or a horizontal orientation, respectively) is superior to performance with stimuli in oblique orientations. Lechelt, Eliuk, and Tanne (1976) and Lechelt and Verenka (1980) first reported similar spatial asymmetries in the haptic modality as well as in visual/haptic cross-modal judgments of stimulus orientations. Lechelt (1988) examined the tactile discrimination threshold for stimulus-orientation discrepancy

This research was supported by the Institute for Perception Research and by Philips Research. The authors wish to thank Peter Goossens and Paul Beerkens for their assistance in developing the control and data-collection software, and Rudi van Hoe for his advice in the application of MANOVA. Address correspondence to David V. Keyson, IPO, P.O Box 513, 5600 MB Eindhoven, The Netherlands (e-mail: keyson@prl.philips.nl). from standard or referent vertical, horizontal, and diagonal reference orientations. The stimuli were vibrating linear displays presented to the pad of the right index finger via an Optacon (optical-to-tactile converter). The Optacon converted optical line images into a $6 \times 24$ tactile array using piezoelectric bimorph reeds vibrating at $230 \mathrm{~Hz}$. Results indicated that deviations of $2.5^{\circ}$ and $5^{\circ}$, respectively, could be discriminated at the $75 \%$-correct level from horizontal and vertical standards, whereas $15^{\circ}$ deviations were required for right and left diagonals. Using the same device and an adaptive-staircase method (Levitt, 1971), Schneider, Hughes, Epstein, and Bach-yRita (1986) also observed an oblique effect and, at the $71 \%$-correct level, found thresholds as low as $7^{\circ}$.

Spatial asymmetries in discrimination thresholds for tactile orientation have been attributed to both neurophysiological and experiential factors (Appelle, 1972). For example, Lechelt (1988) considered the uneven distribution of sensory units in the index finger to be a sensory-neural explanation for the better discrimination of horizontal orientations, while the increased number of rapid-adapting (RA) and slow-adapting Type 1 (SAl) cutaneous receptors in the distal direction (Johansson \& Vallbo, 1976) comprised a sensory-neural explanation for the better discrimination of vertical orientations.

Lechelt (1988) also considered common experience and awareness of the principal meridians as possible contributing factors to tactile spatial asymmetry. Such experiential factors were also examined by Appelle and Countryman (1986), who found that the oblique effect could be significantly reduced for haptic stimuli when subjects were not verbally informed of the test orientations. Given that both the experiential and the neurophysiological factors contribute to the asymmetrical spatial perception of static lines, one might expect directional discrimination of point motion in the vertical and horizontal directions to be superior to that of movements along an oblique axis. 
In considering directional discrimination of moving tactile stimuli, attentional factors may play a role, in particular when more than one stimulus is presented at a time. Gardner and Palmer (1990) found directionsensitive neurons in the primary (SI) cortex of monkeys, suggesting that direction of movement may be registered in a relatively automatic fashion (i.e., without focused attention). However, a recent study by Evans and Craig (1991) suggests that the spotlight theory of attention (Posner, 1980) can be applied to the skin-although, compared with that of the visual system, the spotlight is considerably broader. Selective attention to a moving stimulus, given a simultaneous movement in an incompatible direction, depends to a high degree on the spatial separation between the two stimuli. Results of Evans and Craig's (1991) study indicate that incompatible nontarget movement at an adjacent finger interfered with the subjects' ability to judge direction of movement at a target location, but this was not the case when an incompatible movement was presented to the corresponding finger of the contralateral hand. In addition to spatial separation, the temporal interval between two moving stimuli can affect selective attention. In a recent study by Craig and Evans (1995), the temporal interval between a moving target and a nontarget stimulus, both on the fingerpad, was shown to contribute to errors in directional judgments. In particular, when the stimulus onset asynchronies were reduced from 500 to $52 \mathrm{msec}$, with either target or nontarget presented first, the percentage correct in terms of determining direction of movement was significantly reduced. However, in conditions in which two different directional stimuli were given, with both requiring the same response, the decrease in performance at $52 \mathrm{msec}$ did not occur. Craig and Evans (1995) suggest that response competition may be a major factor in temporal masking.

The velocity and skin site of a moving stimulus have also been shown to influence directional discrimination. The capacity of four neurologically healthy young adults to distinguish opposing directions of cutaneous motion along the upper limbs was considered by Essick, Bredehoeft, McLaughlin, and Szaniszlo (1991). Using a constant-velocity brush, with a traverse length of $0.33 \mathrm{~cm}$, they found optimal directional sensitivity on the index fingerpad to occur at a velocity of $1.5-9.4 \mathrm{~cm} /$ $\mathrm{sec}$, with a mean of $5.4 \mathrm{~cm} / \mathrm{sec}$ across all four subjects. To obtain the fingerpad sensitivity levels measured by $d^{\prime}$ along the forearm, the stimulus had to traverse a distance that was approximately six times longer, and in addition, the velocity of stimulus motion at which direction sensitivity was highest increased systematically, to $18.57 \mathrm{~cm} / \mathrm{sec}$, as the test site was shifted from fingerpad to proximal forearm. Using the same type of device, similar effects on directional-discrimination thresholds, with respect to contact site, were also found by Dreyer, Hollins, and Whitsel (1978), although the optimal velocity range $(3-25 \mathrm{~cm} / \mathrm{sec})$ was the same for arm and wrist. Effects of changes in line length on orientationchange thresholds were also found by Schneider et al.
(1986), who found that subjects were more easily able to discriminate the direction of lines, presented by an Optacon on the left index finger, that utilized the full length, as opposed to half the length of the display. However, while only $2 \%$ of the variation in thresholds was attributed to absolute line length, $28 \%$ was attributed to trials in which a short line was compared with a long line, or vice versa. The velocity of a stimulus has also been shown to affect perception of straightness. As observed by Langford, Hall, and Monty (1973), the accuracy of perception of a straight line formed by a point moving across the volar surface of the forearm or straight track of stimulation increased as the stimulus velocity increased to about $16 \mathrm{~cm} / \mathrm{sec}$.

A limited amount of research has been conducted to consider possible effects of gender and handedness on the perception of tactile direction. In their study using an electromechanical stimulator consisting of three steel rods, Varney and Benton (1975) asked their subjects to judge the direction of stimulation, which was either vertical, horizontal, or along either $45^{\circ}$ diagonal. A main effect for handedness was found $(p<.02)$, whereby $66 \%$ of right-handed subjects showed superior accuracy of recognition in the left hand, while $56 \%$ of the left-handed subjects showed superior accuracy of recognition in the right hand.

Knowledge about the discrimination of a moving tactile stimulus may be used to examine how tactile navigational cues can be communicated to help guide a user through a complex information display. The findings can be further used in the construction of interactive multimodal interfaces, as well as in the development of dynamic tactile input devices. The fingerpad is ideally suited as a point of interface for tactile communication, given the degree of fine motion control that can be exercised, as well as the fine spatial resolution owing to the high spatial density of mechanoreceptors.

An experiment was conducted to measure the ability of subjects to discriminate between directions of a point contact moving across the fingerpad. While it appears that the skin of the fingerpad is sensitive to the difference between forward and backward motion, this experiment was an attempt to clarify (1) to what degree the cutaneous afferents can discriminate directions of motion across a range of orientations for a given contactor size and movement; (2) whether threshold levels are uniform across orientations; and (3) whether angle discrimination for a moving tactile point differs from the tactile discrimination of orientation for a vibrating line contactor. Additionally, pilot experiments were conducted to consider issues raised by reviewers concerning the influence of velocity and temporal masking in the directional discrimination of a moving point stimulus.

\section{METHOD}

\section{Subjects}

To avoid possible handedness factors (Varney \& Benton, 1975) in the perception of moving tactile stimuli, familial right-handed volunteer subjects from IPO were used in the experiment. All of the subjects indicated a strong-to-moderate right-handedness ac- 
cording to the criteria used by Varney and Benton (1975). With participation based upon availability, 14 male subjects with normal or corrected-to-normal vision took part. They ranged in age from 21 to 54 , with a mean age of 30 . With the exception of 2 subjects. none of the subjects had felt the tactile stimuli prior to the experiment.

\section{Apparatus}

A modified version of a trackball with force feedback, developed at IPO, was used to generate a moving point stimulus (Figure 1). A hard-plastic, round, standard-sized braille point, with a diameter of $0.8 \mathrm{~mm}$, a height of $1 \mathrm{~mm}$, and a flat top, was fixed to the ball's surface. The trackball appears as a large gray sphere in the center of Figure 1. The four rubber-rimmed wheels visible around the 56-mm-diam trackball were designed to keep the ball in place. To provide ball forces, two wheels, positioned on the $x$ and $y$-axes, were each attached to a separate servo motor. The two motors appear as long cylinders in Figure 1. The other two adjustable spring-mounted wheels, each opposite a motor wheel, were connected directly to optical position sensors to ensure fine movement cont rol and monitoring of ball position, independent of motor-wheel positions. The control software held deviations from the intended movement path, caused by skin friction, to within $1.5^{\circ}$. Each subject rested his hand in a flat position, perpendicular to the front edge of the trackball, on the 4-mm-thick Plexiglas surface. The right index finger was placed over a single 14-mm-diam hole, such that the point on the ball could be felt in the center of the fingerpad. The hole appears in the inverted triangle over the trackball. The surface around the hole was beveled to accommodate the finger. The entire apparatus was placed inside a cardboard box to conceal possible visual cues of movement direction. The motors did not emit any audible noise. A heating lamp was used to maintain the surface temperature of the device at $30^{\circ} \mathrm{C}$. The subjects were seated alone in a quiet room.

\section{Procedure}

On each trial, two successive stimuli were presented in a $\mathrm{V}$ pattern on the pad of the right index finger, each stimulus consisting of a forward and backward movement from the vertex (Figure 2). On the basis of the results from the initial pilot tests described below, each single movement was $3.25 \mathrm{~mm}$ long and ran for $79 \mathrm{msec}$, at a velocity of $4.1 \mathrm{~cm} / \mathrm{sec}$. One leg of the $\mathrm{V}$ pattern was in a reference direction, while the other, a comparison leg, moved at an angle from the reference direction. The order of stimulus presentation was random, with an equal distribution of reference-first or comparison-first stimulus trials. A $0.5-\mathrm{sec}$ pause was set between reference and comparison movements, and a 1.5-sec pause occurred between trials. After each trial, the subjects reported the

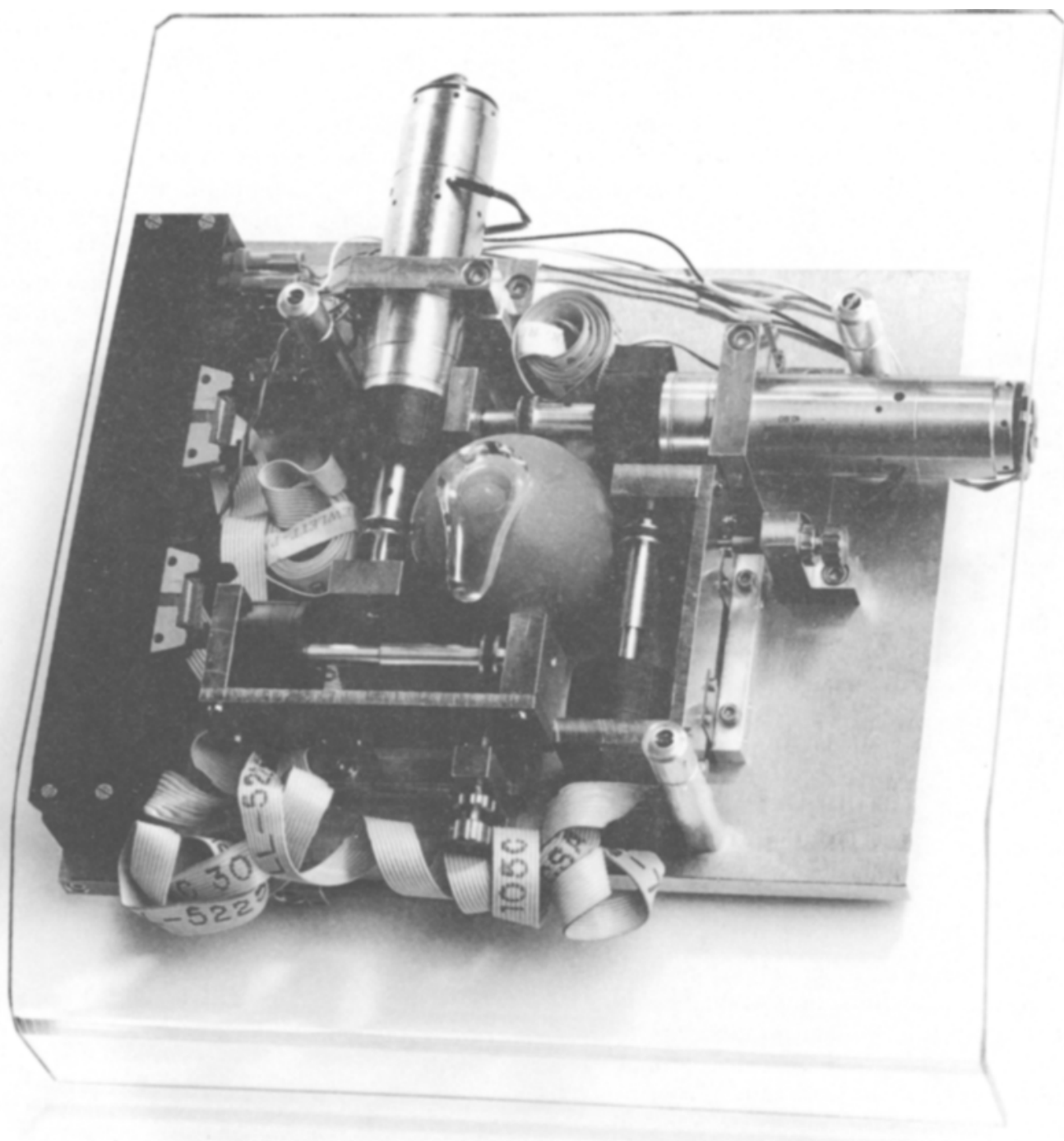

Figure 1. Tactile stimuli device. 


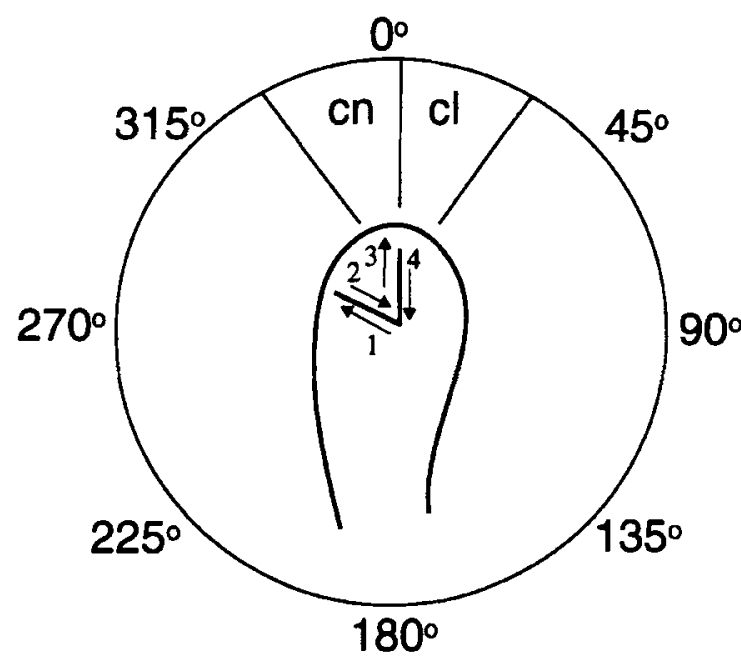

cl: clockwise deviation

\section{cn: counter-clockwise deviation}

Figure 2. Top view of test orientations in relation to index finger of right hand. The four lines drawn on the finger indicate the directions of the point felt under the finger. In this example, the reference orientation is $0^{\circ}$. The comparison movement (Lines 1 and 2) and the reference orientation (Lines 3 and 4 ) were presented in random order, on a trial-by-trial basis. Two correct responses in a row led to a decrease in the angle between the reference and comparison stimuli. An incorrect response led to an increase in the angle.

direction of motion formed by the random order of the two stimuli as being either clockwise or counterclockwise. The left cursor key was used to indicate a counterclockwise direction of motion, and the right key indicated a clockwise motion. Both keys were labeled with a clock-like diagram and an arrow pointing in the appropriate direction. For example, if a reference stimulus at $0^{\circ}$ was given, followed by a comparison stimulus at $150^{\circ}$ (Figure 2), a correct response would be selection of the clockwise key. In the reversed-stimuli order, selection of the counterclockwise key would be a correct response. As described below, two correct responses in a row led to a one-step decrease in the angle between the reference and comparison stimuli. An incorrect response led to a one-step increase in the angle. A correct response followed by an incorrect response was counted as a staircase reversal, as was an incorrect response followed by two correct responses. Once the subject reached 20 staircase reversals, a new measurement was begun from either the clockwise or the counterclockwise side of a reference orientation, for a total of 16 staircases per subject.

Correct judgment of the motion formed by the two stimuli was thus dependent upon discrimination between the reference and comparison stimuli. The orientation under investigation was not readily apparent, thus limiting biases that were due to experiential factors such as those examined by Appelle and Countryman (1986). On a trial-by-trial basis, a low beep tone was given for an incorrect response and a high tone was given for a correct response.

A two-down, one-up adaptive-staircase method (Levitt, 1971) was applied to estimate the $70.7 \%$-correct response threshold for eight reference orientations $\left(90^{\circ}, 45^{\circ}, 0^{\circ}, 315^{\circ}, 270^{\circ}, 225^{\circ}, 180^{\circ}\right.$, and $\left.135^{\circ}\right)$. Using a two-interval, two-alternative forced-choice (2I2AFC) procedure, the angle between the reference and the comparison legs was either increased or decreased, depending on the subjects' response. The following three rules for the two-down, one-up decision criteria were used: (1) Every pair of two consec- utive correct responses causes a one-step decrease in the angle; (2) the angle is increased whenever an incorrect response is given; and (3) after 20 reversals, the staircase run is terminated and threshold is computed as the median value of the last 14 reversals.

Most subjects required about 80 trials to reach the 20 reversals. The initial angle difference between the reference and comparison stimuli was $30^{\circ}$, with a step size of $9^{\circ}$ for the first 3 reversals, followed by $6^{\circ}$ for the next 3 reversals, and $3^{\circ}$ for the last 14 reversals. The first 6 reversals, with larger step sizes, were omitted from the calculation, as they were intended to allow the user to adjust to the experimental procedure prior to actual measurement. As depicted in Figure 2, both the clockwise and counterclockwise sides of the eight reference orientations were evaluated, for a total of 16 staircases per subject. For each staircase, one reference orientation, selected at random, was tested.

The subjects were asked to complete one practice staircase before beginning the experiment. They then completed two staircases per session, with two sessions per day, spread over four days. On average, a staircase took $5 \mathrm{~min}$ to complete. The staircases for each subject were plotted and saved for later analysis. The percentage of correct judgments for the clockwise and counterclockwise stimuli, at each test orientation, was recorded for later analysis of potential response biases.

A series of pilot tests, using 4 subjects, was conducted to optimize the surface height, the diameter of the finger rest hole, the ball displacement, the ball velocity, and the maximum available force level to maintain movement speed, given friction between ball point and finger. Adjustments were made to enable the ball point to maintain contact that could just be perceived along the index pad with minimum skin friction. The finger-hole size was selected to accommodate a wide range of finger sizes, while still being small enough to act as a surrounding reference edge. A maximum-available force level on the ball of 1.5 newtons enabled a constant movement speed of the contact point with the finger in position.

Two additional pilot tests were conducted on the basis of initial feedback from journal reviewers who suggested that directional discrimination thresholds could be lower with a stimulus velocity higher than $4.1 \mathrm{~cm} / \mathrm{sec}$, and that the persistent sensory impression

Table 1

Pilot A: Mean Thresholds for Velocity

(With Standard Deviations) and MANOVA Contrast of Means Analysis

\begin{tabular}{ccccc}
\hline \multirow{2}{*}{$\begin{array}{l}\text { Velocity } \\
\text { (cm/sec) }\end{array}$} & \multicolumn{2}{c}{ Threshold } & & $\begin{array}{c}F \text { Score } \\
\text { of Contrast }\end{array}$ \\
\cline { 2 - 5 } & $M$ & $S D$ & & $d f$ \\
\hline 7.2 & 21.63 & 7.37 & $24.46 \dagger$ & 1,7 \\
4.1 & 14.03 & 4.92 & & \\
1.5 & 14.51 & 5.13 & 0.34 & 1,7 \\
0.5 & 17.86 & 6.14 & $10.17^{*}$ & 1,7 \\
\hline
\end{tabular}

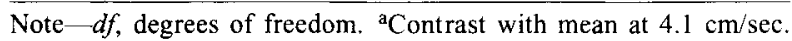
${ }^{*} p<.05$. $\dagger_{p}<.01$.

Table 2

Pilot B: Mean Thresholds (With Standard Deviations) for Interstimulus Interval (ISI, in Milliseconds) and MANOVA Contrast of Means Analysis

\begin{tabular}{rcccc}
\hline & \multicolumn{2}{c}{ Threshold } & & $\begin{array}{c}F \text { Score } \\
\text { ISI }\end{array}$ \\
\cline { 2 - 3 } of Contrast $^{\mathrm{a}}$ & $M$ & $S D$ & $d f$ \\
\hline 2,000 & 15.32 & 6.36 & 1.12 & 1,7 \\
1,000 & 14.88 & 6.77 & 0.84 & 1,7 \\
500 & 13.63 & 5.42 & & \\
50 & 25.01 & 9.91 & $22.44^{*}$ & 1,7 \\
\hline
\end{tabular}

Note- $d f$, degrees of freedom. ${ }^{a}$ Contrast with mean at 500 -msec ISI. ${ }^{*} p<.01$ 
from the first line could alter the perception of the orientation of the second. Using the procedure described above, two groups of 8 right-handed males were assigned to one of two pilot tests. One test, referred to as Pilot A, was conducted to consider the effects of ball velocity on discrimination of direction of motion, while a second test, Pilot $\mathrm{B}$, was performed to consider the delay interval between the comparison and reference stimuli, or interstimulus interval (ISI), as a factor that could reduce any temporal masking effects. In Pilot A, four ball velocities $(0.5,1.5,4.1$, and $7.2 \mathrm{~cm} / \mathrm{sec})$ were used, with an ISI of $500 \mathrm{msec}$. In Pilot B, four ISIs were used $(50,500,1,000$, and $2,000 \mathrm{msec})$, with the ball velocity fixed at $4.1 \mathrm{~cm} / \mathrm{sec}$. In both tests, one of the eight possible orientations was selected at random, with either clockwise or counterclockwise deviations. Over a two-day period, two staircases per treatment condition were run for a total of eight staircases per subject.

The results from the pilot experiments were analyzed using a preplanned multiple analysis of variance (MANOVA) for repeated measures (also known as multiple regression for repeated measures). In repeated measures designs in situations in which assumptions for a traditional analysis are violated, a multivariate analysis is generally accepted as a preferred approach over a univariate test such as an ANOVA. An example of such a situation is one in which there is a time factor in which observations made close together are likely to be more highly correlated than observations separated by longer time intervals (Pedhazur, 1982; Poor, 1973).

The results of the MANOVA indicated main effects for changes in stimulus velocity (Pilot $\mathrm{A}$ ) $[F(3,5)=6.22, p<.01]$ and ISI (Pilot B) $[F(3,5)=5.51, p<.01]$ on mean directional-change threshold. A contrast of means analysis indicated that optimal velocity, in terms of discrimination of direction for a moving point, may not be a single value, but rather a wide range of values from 1.5 to $4.1 \mathrm{~cm} / \mathrm{sec}$ (Table 1). While the low end of this range is lower than the mean optimal velocity for discrimination of a forward versus a backward movement found by Essick et al. (1991), one of the four subjects in the Essick study obtained optimal discrimination at $1.5 \mathrm{~cm} / \mathrm{sec}$. The results of Pilot B confirmed Craig and Evans's (1995) findings regarding the effects of a 50-msec ISI compared with a $500-\mathrm{msec}$ ISI in the discrimination of sequential moving stimuli on the index finger. There was no sign of a persistent sensory impression from the first line that could have altered the perception of the orientation of the second line. Thresholds did not significantly vary as the ISI was increased from 500 to $2,000 \mathrm{msec}$ (Table 2). In short, the pilot tests confirmed the initial settings of a stimulus velocity of $4.1 \mathrm{~cm} / \mathrm{sec}$ and an ISI of $500 \mathrm{msec}$.

\section{RESULTS}

Examination of individual data plots did not show any apparent dependence of directional-change threshold on reference orientation. When the mean of the medianbased estimates across subjects was plotted for each orientation, however, discrimination thresholds with regard to the finger axis were found to be fairly uniform, except for thresholds at $180^{\circ}$.

The calculated threshold for all orientations other than $180^{\circ}$ varied between approximately $12.9^{\circ}$ and $15.6^{\circ}$, whereas the threshold for $180^{\circ}$ was about $10.6^{\circ}$, with both clockwise and counterclockwise deviations being lower in comparison with measurements from the other orientations (Table 3 ). The results of a MANOVA for repeated measures indicated no significant differences between thresholds for clockwise and counterclockwise deviations across the eight reference orientations. The data from the clockwise and counterclockwise sides of
Table 3

Mean Directional Discrimination Thresholds (With Standard Deviations) for Clockwise and Counterclockwise Deviations from Test Orientations

\begin{tabular}{ccccc}
\hline \multirow{2}{*}{$\begin{array}{c}\text { Test } \\
\text { Orientation }\end{array}$} & $M$ & & \multicolumn{2}{c}{ Counterclockwise } \\
\cline { 2 - 3 } \cline { 5 - 6 } & $M$ & & $M$ & $S D$ \\
\hline $90^{\circ}$ & 16.85 & 7.59 & 12.51 & 6.30 \\
$45^{\circ}$ & 13.27 & 5.48 & 13.87 & 4.18 \\
$0^{\circ}$ & 15.35 & 8.11 & 13.88 & 8.83 \\
$315^{\circ}$ & 13.65 & 4.56 & 12.15 & 7.14 \\
$270^{\circ}$ & 14.08 & 5.46 & 17.08 & 5.73 \\
$225^{\circ}$ & 16.76 & 7.66 & 13.76 & 7.17 \\
$180^{\circ}$ & 11.13 & 5.48 & 10.03 & 4.69 \\
$135^{\circ}$ & 16.76 & 8.91 & 14.88 & 8.30 \\
\hline
\end{tabular}

Table 4

Results of MANOVA Contrasts of Means for the Mean of Clockwise and Counterclockwise Mean Discrimination Thresholds at Each Reference Orientation by the Mean of Thresholds at $180^{\circ}$

\begin{tabular}{rccc}
\hline $\begin{array}{c}\text { Orientation } \\
\text { by } 180^{\circ}\end{array}$ & $d f$ & $F$ & $\begin{array}{c}\text { Mean } \\
\text { Difference }\end{array}$ \\
\hline $90^{\circ}$ & 1,13 & $8.79 \dagger$ & $4.10^{\circ}$ \\
$45^{\circ}$ & 1,13 & 3.44 & $2.99^{\circ}$ \\
$0^{\circ}$ & 1,13 & $8.19^{*}$ & $2.56^{\circ}$ \\
$315^{\circ}$ & 1,13 & 1.93 & $2.32^{\circ}$ \\
$270^{\circ}$ & 1,13 & $11.28 \dagger$ & $5.0^{\circ}$ \\
$225^{\circ}$ & 1,13 & $4.79^{*}$ & $4.68^{\circ}$ \\
$135^{\circ}$ & 1,13 & $5.15^{*}$ & $4.44^{\circ}$ \\
\hline
\end{tabular}

aDifference based on $10.58^{\circ}$; mean of the clockwise and counterclockwise discrimination thresholds at $180^{\circ} .{ }^{*} p<.05$. $\dagger_{p}<.01$.

each orientation were subsequently pooled to determine a single threshold estimate for each reference orientation.

A preplanned MANOVA contrast of individual means across orientations (O'Brien \& Kaiser, 1985), treating the thresholds for clockwise and counterclockwise deviations from each orientation as a repeated variable, was conducted to compare discrimination thresholds at $180^{\circ}$ with those of the other seven orientations. The contrast showed that angle discrimination at $180^{\circ}$ was significantly different from that at all of the orientations except $45^{\circ}$ and $315^{\circ}$ (Table 4 ).

No bias was observed in the response for clockwise or counterclockwise stimuli. As expected, percentage correct at threshold level as defined by the 2 I2AFC procedure for both stimuli was about $71 \%$, with $71.48 \%$ correct for clockwise deviations and $71.41 \%$ correct for counterclockwise deviations.

\section{DISCUSSION}

The possibility that our measured directional change thresholds are based entirely on perceived displacement cues cannot be excluded a priori. Had subjects responded to spatial shifts in the endpoints of the reference- and comparison-movement legs (Figure 2), as contrasted to perceived changes in orientation, the predicted minimal discriminable angle would have been about $27^{\circ}\left[2 \arcsin (.75 / 3.25)=26.68^{\circ}\right]$, given that each 
stimulus leg had a length of $3.25 \mathrm{~mm}$, and that the fingertip has a $1.5-\mathrm{mm}$ error of localization (Sherrick \& Cholewiak, 1986). This appears unlikely, given that the average directional change thresholds for the eight major orientations was found to be approximately $14^{\circ}$. However, point localization has been observed to be as low as $0.17 \mathrm{~mm}$ (Loomis, 1979). In an earlier study by Loomis and Collins (1978), using a 1.8-mm-diam waterjet stimulus, excursions $0.1 \mathrm{~mm}$ to the left or right of a reference point were detected correctly $75 \%$ of the time. Given the relationship between point localization and slight movement, as well as the observed effects of stimulus velocity on discrimination thresholds, further research is needed to determine the degree to which endpoint information with little or no motion, as contrasted to constant motion, is used to discriminate the direction of a moving tactile stimulus. Lastly, using variable line lengths as a means with which to control for displacement cues appears to have limitations, as observed in the Schneider et al. (1986) study. The introduction of length changes increased a lowest observed orientation-change threshold from $7^{\circ}$ to $25^{\circ}$.

In comparing observed directional-discrimination thresholds for a moving point with those obtained by Schneider et al. (1986), where the lowest threshold was $7^{\circ}$, the apparently higher thresholds obtained in the current study may be due to differences in the minimum step size used within the adaptive-staircase method (Levitt, 1971). While the lowest step size in the current study was $3^{\circ}$, the lowest measurable angle in the Schneider et al. study was $7^{\circ}$, at a reference orientation of $146^{\circ}$. Since some subjects in the current study were able to detect changes in direction of as low as $3.12^{\circ}$, at a test orientation of $180^{\circ}$, a step size of three was chosen. The larger step size used by Schneider et al. may have reduced the number of trials reaching threshold, thus limiting adaptation to threshold, in addition to reducing the difficulty of discriminating the reference from the comparison stimulus on a trial-by-trial basis. Using the same device, Lechelt's (1988) estimation of orientation-change thresholds for left and right diagonals was $15^{\circ}$.

Apparently, Lechelt's (1988) finding of an oblique effect for apparent vibrating lines does not fully apply to the discrimination of direction for a moving point, given that only a partial oblique effect was found for one of the vertical orientations $\left(180^{\circ}\right)$ that was superior to other orientations. The nature of the tactile stimuli, as well as the method used in eliciting responses, may explain why, in general, no oblique effect was found. A moving-point contactor causes the skin to stretch in the direction of movement, which is not the case with linear vibrotactile contactors, where a static spatial presentation is given. The subjects in Lechelt's study were asked to report which set of two lines (i.e., the first or the second) had an orientation that was not the standard orientation for that condition. The subjects may have been better able to map the tactile stimuli to a familiar spatial model or pattern than were the subjects in the current study, who were required to report whether the lines of motion changed in a clockwise or a counterclockwise direction. In the present study, the reference orientation under investigation was not intuitively obvious.

Lechelt (1988) attributed the lower thresholds for discrimination of deviations from the horizontal orientations, compared with those for the vertical and diagonal orientations, to the increased number of SAI and RA receptors in the distal direction of the index finger (Johansson \& Vallbo, 1976). Increased homogeneity of receptors in the horizontal direction was considered to provide a sensory-neural advantage in the horizontal direction. Such an advantage does not appear to be a factor in the perception of point-contactor movement.

In addition to the spatial distribution of cutaneous afferents in the fingerpad, the stretching of the skin may be a key factor in directional discrimination of pointcontactor movement. In particular, the observed pattern of discrimination thresholds, by orientation, may reflect stretch characteristics of the skin of the index fingerpad. The three cutaneous afferents-RAs, pacinian corpuscles (PCs), and SAs - are triggered in the initial stretch of fingerpad skin, but only the SAs appear to have a significant directional bias in their responses (Westling \& Johansson, 1987; Srinivasan, Whitehouse, \& LaMotte, 1990). Though the mechanism by which the direction of skin stretch is perceived is not clear, Srinivasan et al. (1990) suggest, as one possible explanation, that directional sensitivity of SA neurons may be due to the way fibers innervate specific skin locations. The example given is that the skin in front of a leading edge of a contact area is subjected to parallel compression, whereas the skin behind the contactor area (including the point at which it is anchored to the fingernail) is subjected to tension.

In a classic 1925 monograph by Katz (cited by Krueger, 1982), the relationship between felt friction and movement was discussed. Katz noted that surface hardness-softness, as well as elasticity, is revealed by up-down movement vertical to the material, whereas roughness-smoothness is revealed by lateral movement across the material. In one study, Katz found that the hardness of a pencil was more readily recognized by writing downstrokes than by writing upstrokes. As noted by Krueger, the fingerpads produce different types of forces on inward and outward thrusts. When pushed outward, more resistance is encountered by the fingers, and they move in a jerkier fashion because they tend to dig into the surface.

As a factor of direction of skin stretch, the differences in apparent friction could be due to the fact that the skin is anchored at the fingernail but not at the distal interphalangeal joint. Because of this difference, orientationchange thresholds may tend to be lower at $180^{\circ}$, since tension in the skin can differ significantly where it joins the nail, while one would expect no significant difference in opposite horizontal and diagonal orientations. While the skin near the nail was stretched at $0^{\circ}$, in the second and fourth V-pattern movements, the apparent friction was masked by initial forward compression of the skin.

While the observed pattern of orientation change thresholds appears to reflect stretch characteristics of 
the skin of the index fingerpad, the influence of velocity and temporal factors (Craig \& Evans, 1995; Essick et al., 1991) on directional sensitivity thresholds suggests that in addition to neural activity associated with skin stretch, spatiotemporal factors are key in the discrimination of direction of movement. Essick (1992) attributes more neural activity to spatiotemporal factors rather than to skin stretch on the basis of the fact that reduction in the capacity to recognize the direction of stimulus objects (i.e., directional cutaneous kinesthesia) can accompany peripheral nerve injury, whereas directional dermatokiestheia (i.e., the capacity to appreciate direction of skin stretch) is known to be a less sensitive indicator of neural injury (Essick). Also noted by Essick is unpublished work by Rath and Essick, in which directional sensitivity (i.e., discrimination of forward vs. backward movement), as measured using a vibrating tactile array, was similar to that measured with a servocontrolled brush, as long as the same velocities of motion were delivered and the same chord of skin was traversed. While the encoding of directional information based on movement or on static presentation appears to be similar, one cannot readily assume that skin stretch plays little or no role in directional discrimination of a moving point. As observed in the present study, thresholds appear to reflect the skin-stretch characteristics of the fingerpad. In short, it seems reasonable to hypothesize that information about the direction of stimulus motion is made available by the sequential activation of mechanoreceptors, as well as by simpler mechanisms that do not require spatiotemporal integration.

In conclusion, our results suggest near-uniform discrimination threshold across all orientations, with the exception of $180^{\circ}$. This is despite the differences between individual responses, which may have been due to the way in which the shearing force interacted with the formation of papillary ridges (fingerprints). Overall, orientation-change thresholds for the moving stimuli appeared to be higher than those reported by Lechelt (1988) for nonoblique lines felt along a fixed vibrating line. The oblique effect that they found was generally not apparent in our data.

Future research is aimed at measuring how subjects can replicate the tactile motion, using the tactile display as the response-input device. The results of such work should be used to guide the design of future tactile display for man-machine communication, in particular where there is a need to indicate direction of movement.

\section{REFERENCES}

APPELle, S. (1972). Perception and discrimination as a function of stimulus orientation: The "oblique effect" in man and animals. Psychological Bulletin, 78, 266-278.

APPElle, S., \& Countryman, M. (1986). Eliminating the haptic oblique effect: Influence of scanning incongruity and prior knowledge of the standards. Perception, 15, 325-329.

Craig, J. C., \& Evans, P. M. (1995). Tactile selective attention and temporal masking. Perception \& Psychophysics, 57, 511-518.

Dreyer, D. A., Hollins, M., \& Whitsel, B. L. (1978). Factors influ- encing cutaneous directional sensitivity. Sensory Processes, 2 , $71-79$.

Essick, G. K. (1992). Comprehensive clinical evaluation of perioral sensory function. Oral \& Maxillofacial Surgery Clinics of North America, 4, 503-526.

Essick, G. K., BRedehoeft, K. R., McLaughlin, D. F., \& Szaniszlo, J. A. (1991). Directional sensitivity along the upper limbs in humans. Somatosensory \& Motor Research, 8, 13-22.

Evans, P. M., \& Craig, J. C. (1991). Tactile attention and the perception of moving tactile stimuli. Perception \& Psychophysics, 49, 355364.

Gardner, E. P., \& Palmer, C. I. (1990). Simulation of motion on the skin: III. Mechanisms used by rapidly adapting cutaneous and mechanoreceptors in the primate hand for spatiotemporal resolution and two-point discrimination. Journal of Neurophysiology, 63, 841 859.

JohansSon, R., \& VAllbo, A. B. (1976). Skin mechanoreceptors in the human hand: An inference of some population properties. In Y. Zotterman (Ed.), Sensory functions of the skin with special reference to man (pp. 171-183). Oxford, U.K.: Pergamon Press.

KRUEGER, L. E. (1982). Tactual perception in historical perspective: David Katz's world of touch. In W. Schiff \& E. Foulke (Eds.), Tactual perception: $A$ sourcebook (pp. 1-54). Cambridge: Cambridge University Press.

Langford, N., Hall, R. J., \& Monty, R. A. (1973). Cutaneous perception of a track produced by a moving point across the skin. Journal of Experimental Psychology, 97, 59-63.

LECHELT, E. C. (1988). Spatial asymmetries in tactile discrimination of line orientation: A comparison of the sighted, visually impaired, and blind. Perception, 17, 579-585.

Lechelt, E. C., Eliuk, J., \& TAnNe, G. (1976). Perceptual orientational asymmetries: A comparison of visual and haptic space. Perception \& Psychophysics, 20, 463-469.

LeChelt, E. C., \& Verenka, A. (1980). Spatial anisotropy in intramodal and cross-modal judgments of stimulus orientation: The stability of the oblique effect. Perception, 9, 581-589.

LEVITT, H. (1971). Transformed up-down methods in psychoacoustics. Journal of the Acoustical Society of America, 49, 467-477.

Loomis, J. M. (1979). An investigation of tactile hyperacuity. Sensory Processes, 3, 289-302.

Loomis, J. M., \& Collins, C. C. (1978). Sensitivity to shifts of a point stimulus: An instance of tactile hyperacuity. Perception \& Psychophysics, 24, 487-492.

O'BRIEN, R. L., \& KAISER, M. K. (1985). MANOVA method for analyzing repeated measures designs: An extensive primer. Psychological Bulletin, 97, 316-333.

Pedhazur, E. J. (1982). Multiple regression in behavioral research. Fort Worth, TX: Holt, Rinehart \& Winston.

POOR, D. S. (1973). Analysis of variance for repeated measures designs: Two approaches. Psychological Bulletin, 80, 204-209.

POSNER, M. I. (1980). Orienting of attention. Quarterly Journal of Experimental Psychology, 32, 3-25.

SCHNEIder, S. L., Hughes, B., Epstein, W., \& BACH-Y-Ruta, P. (1986). The detection of length and orientation changes in dynamic vibrotactile patterns. Perception \& Psychophysics, 40, 290-300.

Sherrick, C. E., \& Cholewiak, R. W. (1986). Cutaneous sensitivity. In K. R. Boff, L. Kaufman, \& J. P. Thomas (Eds.), Handbook of perception and human performance (Vol. 1, pp. 23-24). New York: Wiley.

Srinivasan, M. A., Whitehouse, J. M., \& LaMotte, R. H. (1990). Tactile detection of slip: Surface microgeometry and peripheral neural codes. Journal of Neurophysiology, 63, 1323-1332.

VARNEY, N. R., \& BENTON, A. L. (1975). Tactile perception of direction in relation to handedness and familial handedness. Neuropsychologia, 13, 449-454.

WESTLING, G., \& JohANSSON, R. S. (1987). Responses in glabrous skin mechanoreceptors during precision grip in humans. Experimental Brain Research, 66, 128-140.

(Manuscript received February 11, 1994; revision accepted for publication December 20, 1994.) 